\title{
Políticas y gestión de la investigación
}

\section{Scientific policies and research management}

\author{
Manuel Carrasco Mallén \\ Director del Fondo de Investigación Sanitaria. Instituto de Salud Carlos III. Madrid
}

La investigación biomédica es una actividad necesaria para el éxito de cualquier estrategia que se proponga mejorar la salud de los ciudadanos. Teniendo en cuenta las circunstancias que determinan el rendimiento de la investigación, resulta posible el diseño de una cadena de valor de la investigación, que permita identificar las etapas de la misma de manera esquemática y los actores que intervienen. En este artículo se analizan estas etapas desde el punto de vista de las Agencias financiadoras y las Estructuras de investigación, incluyendo los Grupos de investigación. Se hace especial énfasis en el diseño de políticas científicas y en la priorización de la investigación biomédica, concretamente la realizada por el Ministerio de Sanidad y Consumo, basada en la Ley de Cohesión y Calidad del Sistema Nacional de Salud e incluida en el Plan Nacional de Investigación Científica, Desarrollo e Innovación Tecnológica. Desde el ámbito de la gestión de resultados se distingue entre la transferencia y aplicabilidad (Medicina Basada en la Evidencia, Investigación de Resultados en Salud y Programas de Atención Integral de Patologías) y el impacto de la investigación. Por último se describen de forma pormenorizada los Programas del Fondo de Investigación Sanitaria, agencia financiadora del Ministerio de Sanidad y Consumo, concretamente el programa de recursos humanos y difusión de la investigación y el programa de fomento de investigación.

Palabras clave: Investigación biomédica. Cadena de valor de la investigación. Políticas científicas. Prioridades.

Gestión de resultados. Fondo de Investigación Sanitaria.
Correspondencia:

Manuel Carrasco Mallén

Subdirección General de Investigación Sanitaria

Instituto de Salud Carlos III

C/ Sinesio Delgado 8

Madrid

E-mail: carrasco@isciii.es
Biomedical research is a necessary activity for the success of any strategy aiming the improvement of the citizens' health. Bearing in mind the circumstances determining research outcomes, it is possible to design a research chain value allowing to identify in a schematic way its stages and the involved agents. In this article these stages are analysed from the point of view of funding Agencies and research Structures, including research Groups. We place particular emphasis on the design of scientific policies and on the establishment of biomedical research priorities, especially by the Ministry of Health and Consumer Affairs, based on the Unity and Quality Act of the Spanish National Health System and included in the National Plan on Scientific Research, Development and Technological Innovation. From the point of view of the management of outcomes, there is a difference between transfer and applicability (Evidence-Based Medicine, Health Outcomes Research and Programs of Comprehensive Care) and the impact of research. Finally, the Programs of the Health Research Fund, funding agency of the Spanish Ministry of Health and Consumer Affairs are described in detail, especially the program of human resources and dissemination of research, and the program of research promotion.

Key words: Biomedical research. Research chain value. Scientific policies. Priorities. Management of outcomes. Health Research Fund.

\section{INTRODUCCIÓN}

La investigación biomédica es parte fundamental del Sistema Español de Ciencia, Tecnología e Industria. Supone un todo continuo que cubre desde la investigación 
básica fundamental hasta la investigación de la práctica clínica rutinaria, y pasa por fases progresivas de investigación aplicada y clínica, nuevos desarrollos, evaluación (beneficios y costes), y diseminación de los resultados. Además, se trata de una investigación multidisciplinaria, siendo múltiples los factores y circunstancias que participan en la obtención de resultados.

En España, la producción científica biomédica se encuentra localizada en los centros del Sistema Nacional de Salud, incluyendo el Instituto de Salud Carlos III (ISCIII), las Universidades, el Consejo Superior de Investigaciones Científicas (CSIC) y el sector privado.

En los últimos años, la situación de la investigación biomédica ha mejorado de forma notable. El número de investigadores ha aumentado, y la inversión en investigación, desarrollo e innovación (I+D+I) global ha crecido proporcionalmente más que el producto interior bruto. La producción biomédica, comparada con la de otros sectores, también evoluciona favorablemente, de manera que más del $50 \%$ de las publicaciones científicas españolas, presentes en las bases de datos internacionales, corresponden a biomedicina. Nuestro actual desarrollo nos ha permitido pasar de ser meros consumidores de avances, producidos en otros países, a estar entre los generadores de conocimientos científicos.

Sin embargo, esta situación puede ser mejorada ya que nuestra aportación es aun sensiblemente inferior a la de otros países de la Unión Europea. Es deseable que en el futuro inmediato se mantenga y acreciente el esfuerzo inversor, de forma que alcancemos niveles de producción equiparables a los citados países, y el acoplamiento adecuado de los entornos científicos y técnicos con los sectores productivos y financieros.

\section{INVESTIGACIÓN BIOMÉDICA}

La investigación biomédica es una actividad necesaria para el éxito de cualquier estrategia que se proponga mejorar la salud de los ciudadanos. La integración de la investigación con la práctica clínica favorece una mayor calidad de los servicios de salud y una mejor y más rápida implantación de los avances científicos en la prevención, diagnóstico y tratamiento de las enfermedades, así como un cuidado más ético y eficiente de los pacientes. No es posible desarrollar investigación de calidad sin una práctica médica de calidad, y viceversa ${ }^{1,2}$

Dependiendo de sus objetivos inmediatos y las técnicas aplicadas, podemos distinguir diferentes formas o tipos de hacer investigación biomédica ${ }^{2}$ :

- Investigación básica ó preclínica, que persigue el mejor conocimiento de los mecanismos moleculares, bioquímicos, celulares, genéticos, fisiopatológicos, y epidemiológicos de las enfermedades o problemas de salud;
- Investigación clínica (desarrollada en pacientes), cuyo objetivo es el estudio de la eficacia de los procedimientos preventivos, diagnósticos y terapéuticos y el conocimiento de la historia natural de las enfermedades;

- Investigación epidemiológica, en salud pública o servicios de salud (desarrollada en poblaciones), que tiene como objetivo el estudio de la frecuencia, distribución y determinantes de las necesidades de salud de la población, y tratar de conocer la efectividad, calidad y costes de los servicios de salud y la distribución de los recursos.

\section{GESTIÓN DE LA INVESTIGACIÓN BIOMÉDICA: CADENA DE VALOR}

Una vez descrita la actividad de investigación biomédica, veamos como podemos definir su gestión. Buxton ${ }^{3}$ define el rendimiento de la investigación biomédica, haciendo hincapié en el carácter multidimensional de sus resultados. Ello lleva a señalar la diversidad de las valoraciones e intereses correspondientes a los diferentes actores participantes en lo que podemos conceptuar como proceso de la actividad investigadora, y que pasa por las etapas de: priorización, evaluación, selección, financiación, ejecución, difusión y aplicación de la investigación.

En el caso de la investigación biomédica los actores responsables de su ejecución están representados por las agencias financiadoras, las estructuras y centros de investigación, el propio Sistema Nacional de Salud, los pacientes, las empresas relacionadas con el sector, y los investigadores.

Desde el ámbito de los decisores del sistema sanitario y de sus usuarios, el rendimiento de la investigación incluye la noción de coste de oportunidad, y contempla tanto procesos de mejora de la calidad asistencial, como el ahorro potencial de recursos, la mejora en el acceso universal y, especialmente, su contribución significativa a la mejora de la salud. Esta diversidad de valoraciones y preferencias supone, en un entorno participativo con asignación colectiva de recursos, una clara y transparente asignación de prioridades de investigación, y la elección de las alternativas organizativas y los objetivos socialmente más eficientes ${ }^{4}$.

Tenidas en cuenta las circunstancias que determinan el rendimiento, resulta posible el diseño de una cadena de valor de la investigación, que permita identificar las etapas de la misma de manera esquemática y los actores que intervienen $^{3,4}$. (Tabla 1)

La primera etapa, supone el análisis de la situación a través de la identificación y estimación de necesidades de investigación, y el establecimiento de prioridades, con arreglo a criterios explícitos.

Una segunda etapa, de propuestas, evaluación, selección y contrato, refleja el proceso de valoración y negocia- 
Tabla 1. Cadena de valor de la Gestión de la Investigación Biomédica
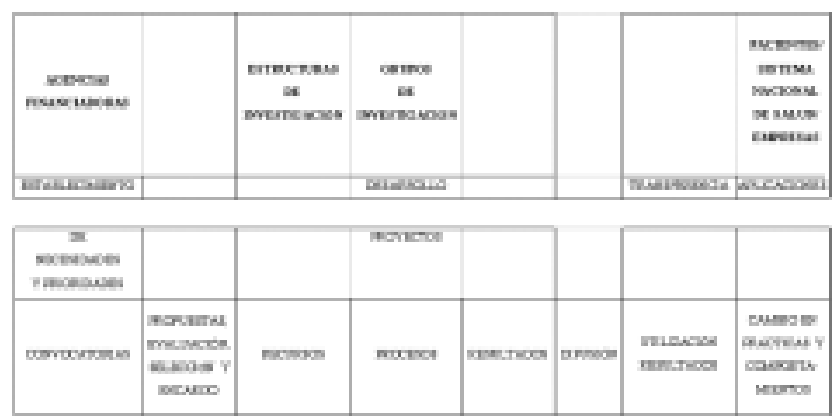

(Elaborado y adaptado a partir del diseño de Buxton, M y Artells Herrero JJ) $)^{3,4}$

ción entre las agencias financiadoras y las estructuras y grupos de investigación en torno a los objetivos y condiciones hechas explícitas por las convocatorias y los intereses investigadores de estos.

La tercera etapa, correspondiente al desarrollo y ejecución de los proyectos que han obtenido financiación, debe considerar la importancia de los recursos, especialmente los intangibles relacionados con la experiencia y competencias profesionales, así como la disponibilidad de una infraestructura tecnológica y procesos adecuados. Del funcionamiento y avance de estos procesos pueden derivarse resultados directos en términos de cambios en la práctica clínica, sin olvidar los resultados primarios cuya valoración y valor añadido deberían tener reflejo a través de los impactos bibliométricos de su publicación y difusión.

La cuarta etapa se refiere a la importancia de las formas de evaluación y difusión de los resultados desde la perspectiva de su aplicabilidad, poniéndose de manifiesto que su mera disponibilidad no asegura la generalización de su conocimiento ni su potencial aportación al sistema sanitario y a la práctica clínica.

Las etapas cuarta y quinta, de transferencia y de aplicación, realzan la importancia de los resultados secundarios, con especial énfasis en la modificación observable de actitudes, comportamientos y prácticas clínicas.

La última etapa, referida al impacto o resultados finales, se refiere al valor añadido de la cadena en términos de indicadores sanitarios poblacionales, siempre que se pueda establecer una asociación entre los resultados obtenidos y la modificación de la salud poblacional.

Como se observa, son diferentes los actores y las interrelaciones que intervienen en el proceso de gestión de la investigación biomédica. El panorama se hace más complejo en los centros hospitalarios debido a la convivencia en estos de tres subsistemas, asistencial, docente e investigador, con predominio del aspecto asistencial en todas las relaciones. Por ello, se hace preciso concebir y diseñar estrategias de investigación apoyadas en elementos de gestión independientes, flexibles, motivadores e innovadores, y adaptados a la realidad de cada centro.

\section{AGENCIAS FINANCIADORAS}

La financiación de la investigación biomédica procede de diversas fuentes, públicas o privadas. Citaremos como principales las siguientes:

- Plan Nacional de Investigación Científica, Desarrollo e Innovación Tecnológica (PNID);

- Planes Regionales de las Administraciones Autonómicas;

- Iniciativas de las Administraciones Locales;

- La Unión Europea (UE) financia investigación a través de:

- Fondos Feder, en regiones objetivo 1 y 2, a través de los programas gestionados por la Administración General del Estado o por las Administraciones Autonómicas,

- VI Programa Marco de Ciencia y Tecnología;

- Iniciativa privada procedente de la industria, fundamentalmente la farmacéutica, fundaciones y asociaciones sin fines de lucro, etc.

\section{DISEÑO DE POLÍTICAS}

La política científica del Ministerio de Sanidad y Consumo se basa en los siguientes principios, señalados en la Ley de Cohesión y Calidad del Sistema Nacional de Salud:

1. Establecimiento de las medidas para que la investigación científica y la innovación contribuyan a mejorar de manera significativa y sostenible las intervenciones y procedimientos preventivos, diagnósticos, terapéuticos y rehabilitadores.

2. Garantía que la actividad investigadora y de transferencia de resultados a la práctica clínica se desarrolla y se sustenta científicamente de manera demostrable.

3. Garantizar la observancia y el cumplimiento de los derechos, la protección de la salud y la garantía de la seguridad de la sociedad, los pacientes y los profesionales involucrados en la actividad de investigación.

4. Incorporación de la actividad científica en el ámbito sanitario en el Espacio Europeo de Investigación.

5. Facilitar que en toda la estructura asistencial del Sistema Nacional de Salud se puedan llevar a cabo iniciativas de investigación clínica y básica, fomentando el desarrollo de la metodología científica y de la medicina basada en la evidencia.

6. Establecimiento de las medidas para que la investigación científica y la innovación contribuyan a mejorar de 
manera significativa y sostenible las intervenciones y procedimientos preventivos, diagnósticos, terapéuticos y rehabilitadores.

7. Garantía que la actividad investigadora y de transferencia de resultados a la práctica clínica se desarrolla y se sustenta científicamente de manera demostrable.

8. Garantizar la observancia y el cumplimiento de los derechos, la protección de la salud y la garantía de la seguridad de la sociedad, los pacientes y los profesionales involucrados en la actividad de investigación.

9. Incorporación de la actividad científica en el ámbito sanitario en el Espacio Europeo de Investigación.

10. Facilitar que en toda la estructura asistencial del Sistema Nacional de Salud se puedan llevar a cabo iniciativas de investigación clínica y básica, fomentando el desarrollo de la metodología científica y de la medicina basada en la evidencia.

Esta política se recoge en la Iniciativa sectorial de investigación en salud que se integra en el Plan Nacional de Investigación Científica, Desarrollo e Innovación Tecnológica 2004-20072.

El PNID 2004-2007 es el vigente instrumento de política científica y tecnológica de la Administración General del Estado Español (AGE), y extiende su ámbito de actuación hasta la innovación tecnológica, proponiéndose impulsar la coordinación con las Comunidades Autónomas y buscar la sinergia con los Fondos Estructurales y el Programa Marco de la UE. La investigación biomédica se contempla específicamente dentro del PNID.

Además, el PNID viene a representar el cumplimiento del Mandato Constitucional que atribuye a la AGE la competencia sobre el fomento y la coordinación general de la investigación científica y técnica.

Por otra parte, los distintos Estatutos de Autonomía han ido estableciendo y desarrollando las competencias que en esta materia posee cada Comunidad Autónoma. La necesaria coordinación, en el campo de la investigación, de las diferentes comunidades entre sí, y de estas con la AGE, se lleva a cabo en el Consejo General de la Ciencia y la Tecnología en el que todos ellos se encuentran representados.

En su diseño el PNDI 2004-2007 identifica los siguientes criterios clave en el área de Biomedicina:

\section{Criterios de carácter científico o tecnológico:}

- Correspondencia con programas similares en otros países, convergencia con otros departamentos y CCAA.

El referente a considerar es el VI Programa Marco de I+D+I (2003-2007) y concretamente el Área Temática denominada "Ciencias de la vida, genómica y biotecnología aplicadas a la salud", que incluye la mayoría de acciones relacionadas con el ámbito de la biomedicina. así como la estrategia para ser partícipes del Espacio Europeo de Investigación. Otros referentes, como modelos, son: el Medical Research Council en Gran Bretaña, el INSERM en Francia y los National Institutes of Health en EE.UU.

- Existencia de investigación de calidad en España:

En los últimos 15 años, España ha conseguido una masa crítica investigadora en el área de Biomedicina y Ciencias de la Salud, en la que ya destacan algunos grupos de investigación de excelencia, entramado para el que deben ejercerse políticas de crecimiento y consolidación. Nuestro desarrollo actual nos ha permitido pasar de ser meros consumidores de avances, producidos en otros países, a comenzar a estar entre los generadores de conocimientos científicos.

\section{- Posición estratégica a largo plazo:}

España es un país que puede competir en igualdad de condiciones, e incluso con ventaja, con algunos países de nuestro entorno, especialmente en algunos campos de la investigación básica y clínica pero la demostración de esta capacidad exige una atención preferente. Para esto es esencial conseguir una mejor coordinación entre la industria farmacéutica y biotecnológica, y centros asistenciales y grupos de investigación de calidad acreditada.

\section{Criterios de carácter económico y empresarial:}

\section{- Volumen de la actividad en I+D en España}

La distribución por campos científicos indica que el 14,3\% del gasto en I+D (INE-2001) se dedica a las ciencias médicas, siendo el campo científico que mayor crecimiento porcentual tiene en los últimos años. A su vez, la Industria Farmacéutica lidera, con el $18 \%$ sobre el gasto total en $\mathrm{I}+\mathrm{D}$, a los restantes sectores industriales de nuestro país.

Además, el $41 \%$ de las publicaciones realizadas por autores españoles y el $51 \%$ de las citas que han recibido estos trabajos corresponden al ámbito de la Biomedicina y Ciencias de la Salud, lo que fundamenta el potencial de nuestro país en este terreno (Mapa de la investigación biomédica - Fondo de Investigación Sanitaria).

Según datos de la National Science Foundation norteamericana, entre 1986 y 1999 la producción científica mundial creció un $14 \%$, con una media de crecimiento anual del $1 \%$. En contraste a estos datos, la producción científica española en Biomedicina y Ciencias de la Salud a lo largo de los últimos veinte años ha crecido a un ritmo medio anual del 12\%, (y del 19\% en cuanto a citas registradas). En este crecimiento, el protagonismo de la investigación procedente de los centros del Sistema Nacional de Salud ha sido muy notorio, al igual que el de las Universidades y Organismos Públicos de Investigación.

Un aspecto que conviene resaltar es el importante papel que los hospitales y centros asistenciales del 
Sistema Nacional de Salud desarrollan en el área de investigación biomédica, dado el importante componente clínico de la investigación

\section{- Grado de dependencia tecnológica}

Las empresas farmacéuticas constituyen un sector consolidado en España, mientras que el sector industrial de productos y equipos médicos está aún muy alejado en sus parámetros de producción e $\mathrm{I}+\mathrm{D}$, de sus homólogos en los países de la UE, lo que ha generado una situación de alta dependencia con el exterior en lo que se refiere a equipos más complejos y de mayor nivel tecnológico.

La globalización del Sector farmacéutico determina una competencia cierta entre los Centros de excelencia distribuidos en los países desarrollados, con frecuentes desplazamientos de la Investigación hacia los lugares más favorables, tanto por el nivel científico o de especialización de tales Centros, como por los incentivos fiscales a la I+D, los menores costes que supone la investigación o la simplificación administrativa que ofrecen tales alternativas.

Por ello, deben potenciarse las iniciativas de carácter público para fomentar la investigación de la industria farmacéutica y biotecnológica en España, con la participación de todos los actores implicados.

\section{- Mejora de la competitividad}

La industria farmacéutica radicada en España cuenta con una tecnología de calidad y alto nivel, y habitualmente colabora con equipos de investigación de centros públicos. Este es un sector que, con el respaldo necesario, permitiría un mayor y mejor desarrollo, si se aproximan las necesidades de la industria a las capacidades de investigación de centros y hospitales, y se mejoran los actuales mecanismos para una buena y rápida transferencia de ideas y tecnología entre ambos sectores. Es de destacar, en este sentido, la reciente creación de parques científicos y tecnológicos.

\section{- Existencia de recursos humanos capacitados}

El número de investigadores biomédicos en España es aún muy bajo, e inferior al de otros países de la UE. Esta situación, claramente deficitaria, condiciona gravemente el desarrollo potencial de este campo. Además, se da la paradoja que el esfuerzo realizado en los últimos años por las distintas administraciones públicas ha dado lugar a una generación de jóvenes investigadores con un elevado nivel de formación y especialización que, sin embargo, tienen dificultades para incorporarse al mercado laboral.

La incorporación de nuevos investigadores plenamente formados es un aspecto esencial para incrementar y posteriormente mantener el grado de calidad deseable en los resultados de la investigación biomédica.

\section{Criterios sectoriales}

Uno de los aspectos distintivos del Programa Nacional de Biomedicina es su carácter sectorial ligado a la política sanitaria. En este sentido hay que tener en cuenta las actuaciones diseñadas para fomentar la investigación de excelencia en el ámbito del Sistema Nacional de Salud, como es el desarrollo de la Ley de Cohesión y Calidad a través de la Iniciativa Sectorial de Investigación en Salud, que contempla entre sus ejes de actuación las siguientes líneas:

- Modernización, fomento de la calidad y competitividad del sistema

- Investigación para la promoción de la salud y la calidad de vida, para una atención a la salud basada en la evidencia, y con un progresivo ajuste hacia las principales patologías de nuestra sociedad; identificando necesidades, problemas y emergencias, reconociendo fortalezas (grupos, infraestructuras) y debilidades; jerarquizando actuaciones según beneficio potencial y viabilidad, además de prestar especial atención a los problemas emergentes y de salud internacional.

- Investigación en salud para la promoción de la competitividad de la industria sanitaria, propiciando un entorno más innovador en los centros sanitarios y contribuyendo al progreso económico y social.

- Medidas y acciones para el fomento de la investigación de transferencia, no solamente la transferencia efectiva de los resultados de las ciencias básicas a la investigación clínica, sino también la del nuevo conocimiento a la práctica clínica y para la toma de decisiones en los servicios sanitarios.

- Intervenciones dirigidas a fomentar una administración más racional y sostenible, que gestiona eficientemente los recursos y que persigue la calidad e integridad en sus actuaciones.

- Aumento de la capacidad y de la coordinación del sistema y de su presencia internacional

- Coordinación y ordenación del espacio español de investigación desde una perspectiva estatal -con otros departamentos y CCAA-, e internacional -Espacio Europeo de Investigación-, con fijación de objetivos y establecimiento de medios específicos, pero complementarios y concurrentes. Extensión y cohesión del sistema a través de la vertebración del Instituto de Salud Carlos III, mediante la creación y acreditación de centros e institutos así como el fomento de alianzas institucionales.

- Actualización de infraestructuras y medidas para el fomento racional, coordinado y sostenible de las grandes instalaciones científicas, servicios comunes y científicotécnicos.

Aumento y actualización de las acciones dirigidas a los recursos humanos, en tanto que eje fundamental del sistema. Se trata de atraer a los jóvenes talentos, favorecer el cambio generacional y, a su vez, desafiar las dificultades y los problemas de motivación de los investigadores clínicos. 


\section{Criterios de interés público: mejora de la calidad de vida y desarrollo sostenible}

La atención sanitaria de calidad constituye una demanda social prioritaria. Un instrumento fundamental para conseguirla es la investigación biomédica. El sector de la salud, como consecuencia de la aparición de nuevas enfermedades y tratamientos, la aparición de las necesidades relacionadas con la mayor esperanza y calidad de vida de la población, y la prestación de servicios que todo ello supone, es un área tecnológica en alza, en la que se prevé un fuerte crecimiento en los próximos años.

Además, es preciso tener en cuenta la progresiva emergencia de epidemias globales, para las que se requieren nuevos escenarios de organización, es decir, una adaptación de los sistemas de investigación de los países desarrollados.

Los problemas de salud y enfermedades responsables de gran morbilidad, mortalidad y pérdida potencial de años de vida productivos, o de gran repercusión social y económica deben ser objeto de interés especial y de la dedicación subsiguiente de recursos para investigación.

Resumidamente, conviene recordar que las características de los Programas Europeos, junto a los criterios señalados en el PNID, hacen necesario que las estrategias para potenciar la investigación biomédica en nuestro país tengan en cuenta los siguientes elementos: el incremento de los fondos económicos; el aumento de la masa crítica; el uso y formación en las nuevas metodologías; la consideración de la investigación de excelencia como un todo, aproximando la investigación básica y clínica; la consideración del hospital como centro de investigación; la priorización y evaluación, fundamentalmente posfinanciación de la investigación; la creación de redes e institutos virtuales (las actuales tecnologías de la comunicación permiten y favorecen el desarrollo de centros virtuales de investigación o redes temáticas, lo que implica la creación de una estructura de coordinación, dotada de los medios adecuados para facilitar esta relación y además, para favorecer la movilidad física del personal científico y tecnológico, así como el establecimiento y gestión de proyectos conjuntos); y la realización de alianzas estratégicas entre centros de investigación públicos y privados.

\section{PRIORIZACIÓN DE LA INVESTIGACIÓN BIOMÉDICA}

La priorización de la investigación biomédica tiene que tener en cuenta los siguientes elementos, tal como muestra la figura:

- la demanda en el SNS y en la sociedad,

- la prospectiva científica y tecnológica a medio/ largo plazo,

- el área de actividad de los grupos de I+D,
- la evaluación de actuaciones nacionales en curso,

- las actuaciones internacionales: VI Programa Marco y construcción del Espacio Europeo de Investigación, y

- la evolución científica y tecnológica del área de biomedicina

Concretamente, la Iniciativa sectorial de investigación en salud que se integra en el Plan Nacional de Investigación Científica, Desarrollo e Innovación Tecnológica recoge las prioridades de la investigación en el Sistema Nacional de Salud.

Para la elaboración de la iniciativa sectorial se han tenido en cuenta las siguientes necesidades y objetivos:

a) Las necesidades de salud de la población y el impulso de la innovación asistencial y la modernización de la estrategia de la $\mathrm{I}+\mathrm{D}$ biomédica, en servicios sanitarios $\mathrm{y}$ de salud pública.

b) La participación de todos los agentes sociales afectados.

c) La transferencia de resultados de investigación, debidamente verificados, a la práctica clínica.

d) La consideración, en su caso, de los resultados científicos en la toma de decisiones por parte de los órganos responsables del Sistema Nacional de Salud.

e) La mejora de la calidad en la gestión de la investigación, mediante la implantación de sistemas eficaces de intercambio de información, evaluación y administración económico-financiera.

f) El impulso, a través del Instituto de Salud Carlos III, del Consejo Superior de Investigaciones Científicas, de otros organismos públicos de investigación y de las uni-

Figura 1

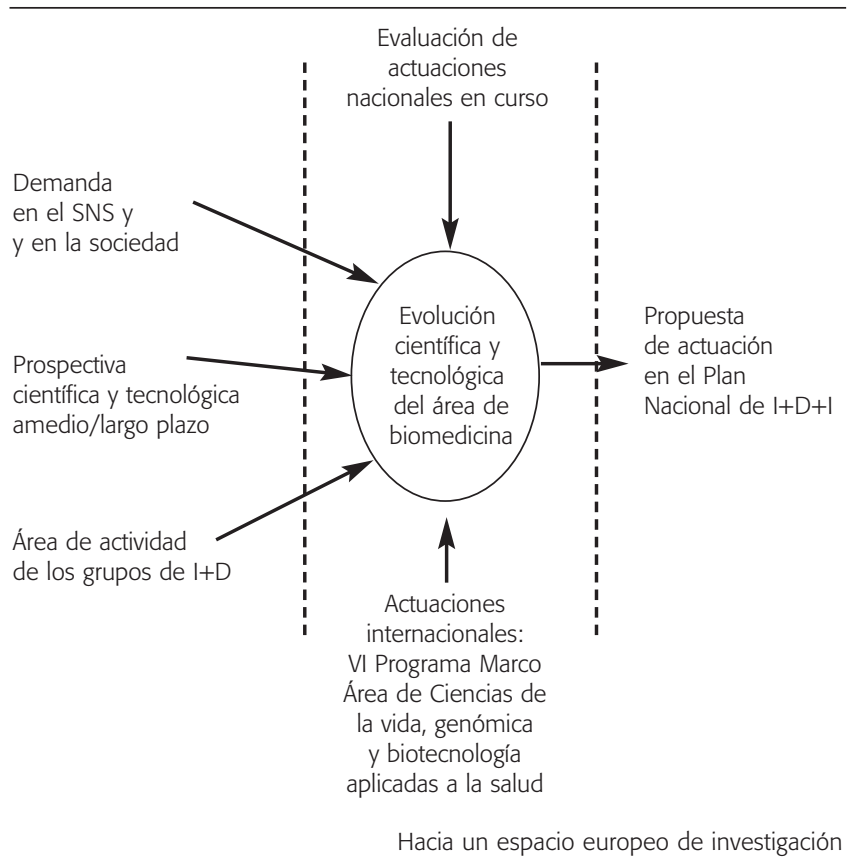


versidades, de la modernización de los centros y redes de investigación del Sistema Nacional de Salud.

g) El incremento de la colaboración con los centros privados de investigación así como con los centros extranjeros, favoreciendo la concurrencia de recursos en aras de objetivos comunes.

h) La conciliación de la actividad investigadora con la normativa vigente y con los principios éticos aceptados por las Instituciones y por la comunidad científica.

Las prioridades identificadas se encuentran ya incluidas en el Plan Nacional, el cual encomienda al Ministerio de Sanidad y Consumo, a través del Instituto de Salud Carlos III, la gestión de los Programas Nacionales de Biomedicina y de Tecnologías para la Salud y el Bienestar.

- Biomedicina: identifica como prioritarios los siguientes programas temáticos:

- Investigación Básica en mecanismos de enfermedad y nuevas estrategias y modelos terapéuticos.

- Investigación Clínica en enfermedades, ensayos clínicos, epidemiología, salud pública y servicios de salud.

- Investigación Farmacéutica en descubrimiento, desarrollo y evaluación de medicamentos.

Estas prioridades se aplican en problemas de salud con mayor carga de enfermedad: Cáncer, Enfermedades Cardiovasculares, Enfermedades del Sistema Nervioso y Mentales, Enfermedades infecciosas y Sida, Enfermedades Genéticas, modelos de enfermedad y terapia, Enfermedades Respiratorias, Otras Enfermedades Crónicas e Inflamación.

- Tecnologías para la salud y el bienestar: identifica como prioritarias las siguientes programas temáticos: Tecnologías de apoyo a las personas con discapacidad y personas mayores, Tecnologías sanitarias e investigación en productos sanitarios y Seguridad y salud en el trabajo. En este caso la investigación evaluativa de tecnologías sanitarias y la investigación en seguridad y salud en el trabajo serán las prioridades a gestionar por el Instituto de Salud Carlos III.

Pero la priorización es un tema complejo, que debe plantearse en coherencia con toda una serie de circunstancias.

La investigación financiada y realizada con fondos públicos participa de las características de bien público. En este caso, el Sector Público interviene en sustitución del mercado como mecanismo de asignación de recursos, debiendo tenerse presente el interés social en la orientación de la investigación.

Para priorizar hay que identificar primero la necesidad, entendida como la desviación o diferencia entre el estado actual o real del conocimiento y la situación considerada como deseable, definida de manera diferente según el punto de vista considerado. Así, podemos distinguir entre:

- necesidades de investigación según el estado de salud de la población: definidas por indicadores, encuestas o métodos de consenso;

- necesidades de investigación expresada por el SNS, de acuerdo a sus políticas, demanda y provisión de cuidados y servicios;

- necesidades emanadas de los investigadores (investigación libre). En este caso, la necesidad definida por expertos (p.e. la comunidad de investigadores) suele prevalecer sobre la preferencia individual como criterio de asignación de recursos ${ }^{3,4}$.

Una vez identificadas las necesidades es preciso identificar criterios de priorización para compararlas. Estos criterios pueden ser diversos:

- la descripción suficiente de la existencia de alguna oportunidad de avance en el conocimiento o en la aportación de evidencia relevante y novedosa aplicable al SNS;

- la constatación de la existencia de una capacidad razonable de los recursos existentes para la consecución de los objetivos establecidos (factibilidad de la investigación);

- la relevancia social y el coste de oportunidad de las alternativas de investigación existentes.

En definitiva el proceso de priorización sigue las siguientes etapas: definición de criterios, estimación y comparación de necesidades de investigación, selección de prioridades de investigación.

Por otro lado, al priorizar en biomedicina es precisa la coordinación con los diferentes programas relacionados (programas marco europeos, nacionales, autonómicos, etc), así como el conocimiento del estado actual de la I+D en las áreas correspondientes, a través de los estudios de prospectiva.

En cualquier caso, deberá ser irrenunciable el mantenimiento de criterios de calidad y eficiencia, sin perder de vista el carácter multidisciplinario y multiinstitucional de este tipo de investigación.

\section{Gestión de la priorización}

Una vez decidido en que se va a investigar, deberá plasmarse esta decisión en los correspondientes programas y convocatorias donde se señalen explícitamente los criterios de priorización que podrán hacerse efectivos a través de fórmulas diferentes:

- contratos temáticos;

- priorización interna en los centros;

- facilitando las economías de escala, el uso compartido de recursos y competencias, y las alianzas estratégicas entre centros de investigación complementarios y sinérgicos;

- desarrollando estrategias que tengan en cuenta las 
nuevas metodologías (genética molecular, biología estructural, bioinformática, investigación de resultados en salud, etc)

\section{Prioridades en investigación biomédica}

En los últimos años varias iniciativas de organismos y comités internacionales se han dedicado a elaborar agendas de investigación en salud o a contribuir para el desarrollo de metodologías para la definición de prioridades. Entre las iniciativas destacan las siguientes ${ }^{5}$ :

El informe denominado "Investing in Health Research and Development", elaborado por el Ad Hoc Committee on Health Research Relating to Future Intervention Options $^{6}$, cuyo objetivo principal fue definir pautas para orientar las inversiones en I y D en salud a niveles nacionales e internacionales. El informe propone como método las siguientes etapas:

a) Identificar la magnitud del problema. A esta interrogante se responde mediante el cálculo de la carga atribuida de enfermedad, condición o factor de riesgo, con base en un indicador denominado años de vida ajustados por discapacidad (AVAD)

b) Identificar las razones para la persistencia de la carga de enfermedad. Ante ello, el comité identifica tres alternativas: a) falta de conocimiento sobre la enfermedad y sus determinantes; b) falta de instrumentos para combatirla; c) deficiente uso de los instrumentos disponibles. Sobre la base de estas alternativas se identifica el tipo de investigación necesaria: si la principal razón es la falta de conocimiento, se precisa más investigación estratégica; si la principal razón es la carencia o el uso deficiente de los instrumentos disponibles, se debe apoyar la investigación operativa.

c) Objetivar si se dispone de conocimientos suficientes sobre el problema para considerar posibles intervenciones. Sobre esta base habrá de actuar la comunidad científica avanzando en el desarrollo de nuevas intervenciones como drogas, vacunas, algoritmos clínicos y políticas. Si por el contrario los conocimientos no fueran los suficientes, pe precisaría más investigación estratégica.

d) ¿Estas intervenciones serán costo-efectivas? ¿Pueden ser desarrolladas a corto plazo y a un costo razonable? El costo-efectividad de una intervención se mide en función del costo por AVAD evitado (se considera atractiva una intervención cuando cuesta menos de 163 euros por AVAD evitado)

e) Objetivar qué se está haciendo actualmente acerca del problema. Se trata de estimar el esfuerzo de investigación actual sobre el problema, a nivel mundial, para valorar si se deben invertir recursos adicionales.

Otra iniciativa a destacar es la del Comité Asesor Global de Investigaciones en Salud (Advisory Committee on Health Research $)^{7}$, organismo asesor de la Directora General de la Organización Mundial de la Salud, integrado por científicos de alto prestigio internacional en diversas disciplinas. En 1998 el comité publicó el informe "A Research Policy Agenda for Science and Technology to Support Global Health Development" El foco principal de la agenda es la contribución de la Ciencia y la Tecnología para el desarrollo de la salud en el ámbito mundial, con el propósito de promover la cooperación entre la comunidad científica, los gobiernos, el sector privado y otros actores. Los principios en los que se basa la agenda incluyen el reconocimiento de que:

a) Existe una ética común a toda la humanidad que permite unificar la acción en pro del desarrollo de la salud, a pesar de la diversidad.

b) Aunque el impacto de la salud es mayormente "local", muchas de sus causas y de sus soluciones son "globales" y multifactoriales.

c) Los desafíos, problemas y determinantes de la salud en el ámbito mundial exigen un enfoque global sistemático para fundamentar acciones a nivel internacional, nacional y local.

d) Los investigadores y las instituciones de investigación deben trabajar conjuntamente en todos los tipos de investigación que contribuyan para la salud.

e) Se deben desarrollar y expandir redes de colaboración para la investigación, aprovechando las ventajas de las nuevas tecnologías de comunicación.

f) Es necesario establecer un proceso continuo de definición, planificación, implementación y evaluación de necesidades y oportunidades de investigación a nivel mundial.

Teniendo como base estos principios, la agenda se concentra en aquellos problemas que son "significativos " y "globales"; los criterios para considerar un problema como "significativo" son los siguientes:

- Contribuye de manera importante -en términos de prevalencia o severidad- en la carga de enfermedad de una población.

- Es percibido como susceptible de ser superado, por lo que genera presiones políticas para su corrección.

- Representa un alto costo para los sistemas de salud.

- Puede transformarse en un riesgo importante para la salud y el bienestar general.

Un problema "significativo" se considera "global" cuando:

- Afecta a poblaciones de varias regiones.

- Afecta a poblaciones de una o de pocas regiones, pero potencialmente puede afectar varias más.

- No puede ser resuelto por una sola región.

- Está limitado a una región, pero los resultados de investigación son generalizables a varias regiones. 
- Exige un tipo de I y D capaz de asumir un alto valor paradigmático como, por ejemplo, en términos metodológicos.

Los macrodeterminantes de importancia crítica para la salud a nivel global que la agenda identifica y analiza son los siguientes:

- Dinámica poblacional.

- Industrialización y urbanización.

- Deterioro ambiental.

- Disponibilidad inadecuada de agua y alimentos.

- Enfermedades emergentes y reemergentes.

- Problemas sociales y de comportamiento.

A partir de lo que se designa como los cinco principales "territorios" de la salud humana (condiciones de salud/enfermedad; sistema de atención; alimentación y nutrición; ambiente y características socioculturales), la agenda identifica los imperativos y las oportunidades de investigación, tanto entre los territorios como hacia el interior de los mismos.

Después de analizar los avances de la ciencia en diversas disciplinas y tipos de investigación, la agenda plantea los desarrollos metodológicos que se requieren, identifica el papel de los principales actores involucrados en las actividades de ciencia y tecnología, y finaliza con la propuesta de creación de redes inteligentes de investigación o IRENES, del inglés Intelligent Research Networks. Las IRENES, basadas en las nuevas tecnologías de información y comunicación, constituyen la principal estrategia para la implementación de la agenda al permitir la comunicación permanente entre la comunidad científica en el ámbito global, facilitando el seguimiento del estado del arte del conocimiento, ayudando a definir la mejor manera para su utilización y la identificación de los lugares dónde los nuevos conocimientos son necesarios, y orientando nuevas iniciativas de investigación.

La tercera iniciativa que queremos destacar es la promovida por el Centro Internacional de Investigaciones para el Desarrollo (International Development Research Centre) de Canadá, con apoyo del Council on Health Research for Development (COHRED) y de la Organización Panamericana de la Salud. Esta agenda, denominada "Prioridades en la investigación de la salud colectiva en América Latina", se concentra en los problemas de la región y en el papel de la investigación en salud pública (o salud colectiva)

En este caso, un grupo de expertos, a través de técnicas de consenso, buscaron identificar grandes tendencias sociales, políticas y económicas de la región y sus relaciones con el proceso salud, enfermedad y asistencia sanitaria, poniendo de manifiesto un déficit de conocimiento sobre estas relaciones.

Se prestó especial atención a determinadas tendencias poblacionales, como la transición demográfica y epidemiológica; el incremento de la pobreza y de la inequidad; los cambios en los estilos de vida y la estructura familiar; el deterioro ambiental, y la reforma de los sistemas asistenciales. Para cada una de ellas se procuró analizar la información y el conocimiento existentes sobre la magnitud del problema y sobre sus implicaciones para la salud, indicando las lagunas existentes y las posibles líneas de investigación. Se incluyó también un capítulo sobre la situación de la investigación en la región y la capacidad de respuesta de su infraestructura científica ante las necesidades de investigación identificadas. Sin embargo, no se incluyeron estrategias de implementación de las prioridades propuestas.

En su conjunto, las tres iniciativas representan un importante avance en términos de metodologías de definición de prioridades. Sin embargo su destino común fue haber constituido documentos interesantes, sin lograr consolidarse como guías para la acción de organismos de apoyo a la investigación nacionales e internacionales. Esto podría ser el resultado de que su elaboración estuvo básicamente a cargo de miembros de la comunidad científica, sin la participación de otros actores. En otras palabras, prevalecieron los aspectos técnicos en detrimento de los aspectos políticos del proceso de definición de prioridades.

A pesar de esa limitación, iniciativas como estas pueden ser importantes instrumentos metodológicos de apoyo a los procesos de priorización con una actitud más proactiva en la promoción de la investigación. Se pone de manifiesto como en lugar de la postura tradicional de investigación libre en espera de la iniciativa espontánea de los investigadores, se avanza hacia la búsqueda de modalidades, inducidas a través de concursos, convocatorias y proyectos multicéntricos, orientadas por agendas de prioridades construidas con amplia participación social ${ }^{5}$.

\section{Gestión en las Agencias financiadoras}

Es necesario incrementar los fondos públicos y privados destinados a la investigación, promoviendo la participación de los sectores industriales, asociaciones, fundaciones, etc.

Para conseguirlo, las Agencias financiadoras deberían cumplir criterios como:

- aplicar principios de transparencia y rigor;

- hacer las fuentes de financiación más próximas, flexibles, amplias, y complementarias compartiendo bases de datos y sistemas de información;

- fomentar el acceso a la financiación de todos los sectores;

- introducir fórmulas de gestión eficaces: feed-back con los centros receptores para eliminar barreras de entrada a la investigación; 
- establecer y dar publicidad a los objetivos estratégi$\cos ^{9}$;

- informar sobre el comportamiento de su gestión y la evaluación de sus infraestructuras, procesos y resultados.

Entre sus funciones las agencias financiadoras deberían:

- procurar la financiación sin recortes del presupuesto solicitado entre los proyectos evaluados positivamente, siempre que sea razonable;

- justificar las modificaciones del presupuesto y dar instrucciones sobre su redistribución;

- fijar criterios sobre diversos capítulos del presupuesto del proyecto: becarios, contrataciones laborales, personal para el trabajo de campo, material inventariable, fungible, viajes, etc;

- posibilidad de financiar proyectos relacionados y por periodos de tiempo más amplios;

- continuar y aumentar la cultura y obligación de "dar cuenta" de la ejecución presupuestaria y científica del proyecto de investigación;

- realización de auditorías in situ y concienciar de la importancia de la investigación a los gerentes de los centros.

\section{ESTRUCTURAS DE INVESTIGACIÓN}

Para obtener los resultados apetecidos, las estructuras de investigación biomédica deben conjugar los siguientes elementos: una estrategia, unos recursos (económicos, infraestructuras científicas y personal investigador), y una gestión adecuada.

La estrategia de los centros de investigación sanitarios (hospitales) debería tener como objetivo aumentar su competitividad en el sector de la investigación, mediante la interrelación y coordinación de sus subsistemas (asistencial, docente e investigador) y los grupos de investigación con el fin de conseguir sinergias y economías de escala. Esta estrategia debe tener en cuenta dos elementos: en primer lugar la especificidad de los activos de las estructuras de investigación biomédica (infraestructuras científicas sometidas a una rápida obsolescencia, e investigadores con la necesidad de mantener su competencia profesional), y en segundo lugar, la relación existente entre una práctica médica altamente cualificada y la realización de investigación de calidad, y viceversa.

La estrategia elegida se debería apoyar en una actitud de liderazgo y compromiso de los responsables de los centros, una organización y dirección científica única, la existencia de una estructura horizontal y común de apoyo a la investigación, la priorización de las líneas de investigación en función de la relevancia para el centro, el SNS y el propio entorno o contexto social, la existencia de una evaluación externa de las actividades de investigación, y el impulso de la transferencia y aplicación de los resultados a la mejora de la práctica clínica.

Sería deseable que este esfuerzo se completase con la realización de alianzas con otros centros y organismos, para fomentar la creación de redes de investigación o unidades mixtas cooperativas, que facilitarían la calidad de los servicios, la motivación de su personal, la innovación en sus actividades, el atractivo para el sector público y privado, y la apertura a la sociedad en general, al SNS, a otros organismos públicos de I + D y al sector privado.

Hay que conseguir cada vez más que los centros sanitarios gestionen la investigación biomédica de manera diferenciada, al igual que lo hacen con la actividad asistencial $^{10}$. La investigación es un producto específico y complejo dentro del hospital que necesita ser gestionado. Este tipo de actividad con frecuencia consume recursos de los centros, que no se identifican como tales (se estima que el efecto de la docencia e investigación varía entre un 7 y un $15 \%$ de los costes de los hospitales universitarios) $)^{11,12}$. La gestión diferenciada de la investigación puede añadir transparencia al proceso, mejorando la cultura de dar cuenta del empleo de los recursos. Igualmente debe, a través de la mejora de los procedimientos de planificación, aumentar la capacidad de los centros para captar fondos de investigación y rentabilizar los ya existentes que, en muchos casos, son obtenidos mediante procedimientos en régimen de concurrencia competitiva. A la vez, esta gestión específica puede estimular y facilitar la participación en convocatorias de agencias financiadoras, la realización de actividades a través de convenios y contratos, y la suscripción de alianzas estratégicas con centros públicos y privados.

La gestión diferenciada debe incorporar sistemas de registros y de apoyo tales como una contabilidad específica, planificación presupuestaria, auditoría externa y desarrollo de un manual de procedimientos y buenas prácticas de investigación.

Como nuevas fórmulas de gestión se están promoviendo fundaciones de investigación, institutos u otras estructuras específicas, para aumentar la autonomía, la flexibilidad y la agilidad ${ }^{10}$. Estas fórmulas deberán dotarse de estructuras de soporte administrativo que faciliten la función prevista, con frecuencia compleja.

Resulta recomendable la formulación de contratos de gestión entre los grupos investigadores y la dirección de investigación, y entre los gerentes y los Servicios de Salud respectivos, con el fin de estimular la autonomía, la motivación y corresponsabilidad de los investigadores y gestores.

\section{GRUPOS DE INVESTIGACIÓN}

Considerados los grupos de investigación, como conjuntos de científicos que se reúnen y trabajan por un obje- 
tivo común, y elementos fundamentales de la estructura investigadora, la gestión debe favorecer su desarrollo y actividad a través de:

- el incremento en sus dotaciones y masa crítica, fundamental para la obtención de resultados;

- la interrelación y comunicación entre grupos o centros;

- el apoyo a la formación del personal investigador;

- la motivación tanto extrínseca como intrínseca, incluyendo el desarrollo de una carrera profesional y el estímulo de políticas de incentivos;

- la planificación del personal investigador y de apoyo a la investigación, incluyendo el personal contratado con cargo a proyectos de investigación, tanto con financiación propia como externa;

- el diseño de una política de becarios que se refleje explícitamente en un documento de incorporación y evaluación continua.

Sería conveniente coordinar los grupos que estudian los mismos problemas y financiar los más rentables manteniendo un equilibrio con los que comienzan o tienen menor experiencia; estimular la búsqueda activa de talentos y jóvenes investigadores.

\section{GESTIÓN DE RESULTADOS}

La gestión de resultados es una de las últimas etapas de la cadena de valor. Su difusión, desde la perspectiva de la disponibilidad de los mismos, se gestiona a través de la producción científica, valorada, a su vez, por los diferentes índices bibliométricos.

Comentaremos brevemente dos aspectos al respecto, la transferencia y aplicabilidad, y el factor impacto.

\section{Transferencia y aplicabilidad}

A través de estos conceptos, se pone de manifiesto la importancia de los resultados secundarios con especial énfasis en la modificación observable de actitudes, comportamientos y prácticas clínicas.

La aplicación de la investigación clínica en la práctica se traduce a lo que hoy se conoce como Medicina Basada en la Evidencia $(\mathrm{MBE})^{13}$, que podemos definir como la utilización consciente y juiciosa de la mejor evidencia, derivada de la investigación clínica, para tomar decisiones en el cuidado de los pacientes. De otra forma, se conceptúa como la definición de criterios para la toma de decisiones clínicas basados en datos de eficacia sobre las distintas opciones de tratamiento posibles. La MBE, al basarse en datos de eficacia se mueve en el entorno de lo que podríamos denominar una práctica clínica ideal, objetivo de la Investigación clínica.

No obstante, esas condiciones de práctica clínica ideal son difíciles de reproducir por lo que es necesario avanzar en medir resultados en condiciones de práctica clínica habitual. Esta iniciativa que se conoce como Investigación de Resultados en Salud ${ }^{13}$ es una actividad multidisciplinar que utilizando métodos de investigación experimentales u observacionales mide los resultados de las intervenciones sanitarias en condiciones de práctica clínica habitual o efectividad.

La Investigación de Resultados en Salud examina las consecuencias derivadas de las intervenciones, identifica los determinantes de las diferencias entre la eficacia y la efectividad, identifica las barreras para el ejercicio de la mejor medicina práctica posible basada en las evidencias disponibles. Es una actividad de investigación que evalúa las consecuencias de las decisiones tomadas de la utilización consciente y juiciosa de la mejor evidencia, derivada de la investigación clínica, en la práctica clínica habitual.

$\mathrm{Su}$ necesidad se justifica por la aparición de nuevas intervenciones sanitarias, el aumento de los costes sanitarios, la variabilidad en la atención sanitaria recibida por los pacientes, la participación creciente de los pacientes en la toma de decisiones médicas, y la utilización de nuevos sistemas de información en la medicina clínica.

Todo ello conlleva la necesidad de realizar estudios para evaluar la relación coste efectividad; el desarrollo de Guías de Práctica Clínica que faciliten criterios para la toma de decisiones clínicas junto con los resultados de los estudios de coste-efectividad antes mencionados; y el empleo de variables de resultados tales como la calidad de vida, la satisfacción con el tratamiento y otras variables relevantes para el propio paciente.

Por otro lado, la existencia de nuevas tecnologías y sistemas de información y la aplicación general de la informática, hace que se pueda tener acceso fácil a los resultados clínicos y económicos de una gran cantidad de pacientes.

La Investigación de Resultados en Salud analiza los resultados obtenidos por la $\mathrm{MBE}$ en condiciones de práctica clínica habitual, analiza la efectividad de las intervenciones sanitarias, hace énfasis en los beneficios del paciente, utiliza métodos de investigación establecidos, y puede tener un enfoque individual (paciente) y/o agregado (poblacional).

La aplicación de la Investigación de Resultados en Salud en la práctica clínica se traduce en los conocidos Programas de Atención Integral de Patologías (PAIP) ${ }^{13}$, cuyo objetivo es la organización y gestión de enfermedades y servicios sanitarios.

\section{Impacto}

Supone la identificación del valor añadido en términos de beneficio en indicadores sanitarios poblacionales, permitiendo establecer asociaciones de las ganancias en los 
indicadores con los resultados de la investigación ${ }^{4}$. Se trata de un concepto donde hay mucho que explorar y avanzar y que debe ser considerado desde las agencias financiadoras en la evaluación posfinanciación de sus programas ${ }^{14}$.

Igualmente debe avanzarse en la elaboración de indicadores y medidas que puedan utilizarse para la evaluación del Impacto Social de la Investigación ${ }^{15,16}$.

Todas estas actividades deben ser potenciadas en el SNS, debiendo disponerse por parte de las Agencias financiadoras los recursos e instrumentos que favorezcan la gestión de resultados con el objeto de evaluar sus propios programas de apoyo y fomento de la investigación.

Por otro lado los centros de investigación deben estímular la actividad de las Oficinas de Transferencia de Resultados de Investigación (OTRI), como interfaz con el mundo empresarial y de la innovación.

\section{PROGRAMAS DEL FONDO DE INVESTIGACIÓN SANITARIA}

La agencia financiadora de la investigación del Ministerio de Sanidad y Consumo es el Fondo de Investigación Sanitaria (FIS), el cual está incluido en la estructura del Instituto de Salud Carlos III. Actualmente, aproximadamente el 70\% de los recursos del FIS se destinan a la financiación de actividades de investigación en centros del Sistema Nacional de Salud, hospitales principalmente, a través de convocatorias públicas en régimen de concurrencia competitiva.

Los Programas del Fondo de Investigación Sanitaria son el Programa de Recursos Humanos y Difusión de la Investigación y el Programa de Fomento de la Investigación , que se describen a continuación:

\section{Programa de recursos humanos y difusión de la investigación}

Tiene como objetivo la potenciación de los recursos humanos en I+D+I en el SNS así como la divulgación y difusión de los resultados de la investigación biomédica.

Este Programa tiene las siguientes actividades:

- Becas de formación en investigación (BEFI) ${ }^{17}$

$\mathrm{Su}$ objeto es desarrollar la formación de investigadores que sirvan de nexo de unión entre la investigación básica, clínica y epidemiológica en el campo de la investigación biomédica.

- Becas de ampliación de estudios $(B A E)^{17}$

$\mathrm{Su}$ objeto es potenciar la formación y capacidad investigadora de los profesionales del SNS, mediante la subvención para cubrir los gastos de viajes y estancias en instituciones nacionales o extranjeras, que permitan la incorporación al SNS de procedimientos asistenciales o de investigación y posibiliten una constante actualización de la acción sanitaria.

\section{- Becas de formación en investigación} para enfermería ${ }^{17}$

Su objeto es impulsar la investigación clínica de enfermería en el Sistema Nacional de Salud, en aplicación del Convenio marco de colaboración suscrito entre el Instituto de Salud Carlos III y la Universidad de Montreal, que tiene como objetivo la formación de profesionales de enfermería en habilidades de investigación con el fin de que a su regreso sirvan de facilitadores y promotores de la investigación en enfermería en su ámbito de influencia.

- Becas de Gestión de Investigación ${ }^{17}$

$\mathrm{Su}$ objeto es promover la formación en los distintos ámbitos de la gestión de la investigación, incluyendo la coordinación, seguimiento y evaluación de las ayudas y programas de investigación biomédica.

- Becas FIS -Fulbright- Sector privado ${ }^{18}$

$\mathrm{Su}$ objeto es introducir el Programa Fulbright en el ámbito sanitario, fundamentalmente en aspectos de gestión y economía de la salud.

Este programa cuenta con la cofinanciación de la industria farmacéutica.

\section{- Contratos de investigadores ${ }^{19}$}

$\mathrm{Su}$ objeto es incrementar, mediante la cofinanciación con los centros, los recursos humanos de calidad en los grupos de investigación del Sistema Nacional de Salud, incorporando a los mismos los conocimientos y la experiencia adquirida en instituciones nacionales y extranjeras por Doctores y otros profesionales con acreditada trayectoria investigadora en Biomedicina.

Esta convocatoria llevará unida la financiación de un proyecto de investigación para el investigador seleccionado.

Además por parte del Instituto de Salud Carlos III se ha creado un fichero de datos de carácter personal (Registro de investigadores biomédico ${ }^{20}$ ) para facilitar la incorporación de investigadores a los diferentes Centros del Sistema Nacional de Salud.

- Contratos de apoyo a la investigación ${ }^{21}$

$\mathrm{Su}$ objeto es ofrecer otras modalidades de cofinanciación para contratar aquellos recursos y servicios de apoyo a la investigación que los centros necesiten de acuerdo a sus necesidades.

- Programa piloto, para contratar profesionales sanitarios que hayan finalizado el período de Formación Sanitaria Especializada ${ }^{22}$

Su objeto es contratar profesionales sanitarios a través de la cofinanciación con las Sociedades Científicas, Universidades, Fundaciones, Hospitales, Atención Primaria, que hayan finalizado el período de Formación 
Sanitaria Especializada, con el objeto de completar su formación en Investigación Biomédica y en Ciencias de la Salud

\section{- Acciones especiales ${ }^{17}$}

Su objeto es contribuir a la más amplia difusión de las innovaciones científicas y técnicas en el campo biomédico, de acuerdo a las directrices del Plan Nacional de Investigación Científica, Desarrollo e Innovación Tecnológica 2000-2003, a través de la financiación de reuniones científicas, publicaciones científicas y publicaciones científicas periódicas españolas en diferentes soportes.

\section{Programa de fomento de investigación}

Tiene como objetivo fomentar la investigación científica en disciplinas biomédicas para así contribuir a fundamentar científicamente los programas y políticas del Sistema Nacional de Salud, en coordinación con los objetivos y criterios establecidos en el Plan Nacional de I+D+I 2000- 2003.

Este Programa tiene las siguientes actividades:

- Proyectos de investigación $n^{23}$

$\mathrm{Su}$ objeto es promover, en el ámbito de las ciencias de la salud, investigación de carácter básico, clínico, clínicoexperimental y de salud pública en las instituciones de Sistema Nacional de Salud, en Universidades y en Organismos Públicos de Investigación, para contribuir a fundamentar científicamente los programas y políticas del Sistema Nacional de Salud. Todas las propuestas de proyectos de investigación deberán adecuarse a los objetivos y criterios establecidos en el Plan Nacional de I+D+I 2000-2003, en el marco del área científico-tecnológica de Biomedicina, del área sectorial Sociosanitaria y de la acción estratégica de Telemedicina. Se introduce, entre otras, la investigación en prevención de riesgos laborales y la investigación en enfermedades raras y poco frecuentes, en atención primaria y en enfermería.

Los proyectos de investigación de carácter básico tendrán una orientación hacia el paciente, siendo requisito necesario para su financiación la participación en los mismos de Centros del Sistema Nacional de Salud con el objeto de desarrollar una estrategia que aproxime la investigación básica y clínica y potencie el concepto del Hospital como centro de investigación.

\section{- Infraestructuras de investigación ${ }^{24}$}

Su objeto es dotar a los Centros del Sistema Nacional de Salud de instalaciones y equipos instrumentales de uso comunitario ó compartido por distintos grupos de investigación, cuyo destino se dirige a las Unidades de Apoyo o Soporte a la Investigación ó estructuras organizativas similares con el fin de ocupar vacíos tecnológicos dentro de la investigación y evitar duplicidades. Las Unidades de
Apoyo o Soporte a la Investigación o estructuras similares deben desarrollarse según las necesidades de cada centro de Investigación, sin que pueda establecerse un modelo único. Sus miembros deben, además de desarrollar proyectos de investigación propia en ámbitos de su competencia, proporcionar a todos los investigadores distintos tipos de apoyo y soporte: apoyo y soporte a aquellos aspectos metodológicos o instrumentales y de servicios comunes, de interés para varios equipos o líneas de investigación del centro (asesoramiento en el diseño, conducción, control de calidad y análisis de datos, asesoramiento en aspectos éticos y legales de los proyectos); soporte a procedimientos comunes (estabulario, bancos de tejidos, criopreservación, análisis de imágenes, laboratorios de soporte a la investigación) y soporte en relación con las tecnologías de la información (bibliotecas, bases de datos bibliográficos, comunicaciones).

\section{- Investigación Evaluativa de Tecnologías Sanitarias $^{25}$}

La Evaluación de las Tecnologías Sanitarias ha venido configurándose en los últimos años como una disciplina fundamental y estratégica para el desarrollo y mantenimiento de los Servicios de Salud a través del logro de un uso más efectivo y eficiente de los recursos sanitarios disponibles.

Su misión es proporcionar información fiable, sintética y clara para la toma de decisiones en los diferentes niveles profesionales, administrativos y políticos que operan en el Sistema Nacional de Salud. El logro de tal misión depende de la realización de estudios e investigaciones dirigidas a responder preguntas especificas que el propio SNS se haya planteado en el ejercicio de sus funciones planificadoras, reguladoras y asistenciales.

En este sentido, a lo largo de los últimos años se ha desarrollado en el seno del Consejo Interterritorial del Sistema Nacional de Salud un proceso de identificación y priorización de tecnologías evaluables. El resultado de ese proceso ha sido una lista priorizada de tecnologías sanitarias, asociadas en determinados casos a condiciones clínicas específicas, sobre las que existen incertidumbres que se materializan en preguntas concretas de investigación de orientación evaluativa que se incluyen en esta convocatoria. Así el objeto de esta acción es promover y fomentar la generación de información y conocimiento relevantes relativos a tecnologías sanitarias de interés para el Sistema Nacional de Salud a través de la financiación de proyectos específicos de investigación de carácter evaluativo.

\section{- Redes de investigación ${ }^{26}$}

El objeto de esta acción es la creación de redes de investigación cooperativa de Centros y grupos de Investigación Biomédica que posean una importante masa crítica de cien- 
tíficos que sean de tipo multidisciplinario y participados por distintas instituciones estatales y autonómicas, con el objetivo de realizar proyectos de investigación cooperativa de interés general. Las redes se constituyen por la asociación de Centros y Grupos de investigación de diferentes Administraciones, Instituciones y Comunidades Autónomas, del sector público o privado con líneas y objetivos de investigación común con el objeto de promover la complementariedad de actuaciones compartiendo objetivos y recursos. Con esta tipología de redes se pretende la creación de esquemas de cooperación científica más potentes que permitan alcanzar objetivos que difícilmente podrían plantearse en un contexto de ejecución más restringido.

La creación de redes de investigación priorizará la investigación de calidad como un todo, desde la investigación básica a la de salud pública. Igualmente se primará la participación de grupos emergentes como integrantes de la red. Se pretende aumentar la masa crítica y la rentabilidad en aquellas áreas prioritarias en el Plan Nacional, en el ámbito sanitario.

\section{- Unidades Mixtas y Centros asociados}

El objeto de esta acción es promover mediante convenios, de acuerdo al artículo 4 apartado a) del Estatuto del Instituto de Salud Carlos III, aprobado por Real Decreto 375/2001, de 6 de abril, la creación de centros asociados y unidades mixtas de investigación y/o formación con instituciones de carácter público o privado, para el desarrollo efectivo de las funciones del Instituto de Salud Carlos III.

\section{- Investigación en Enfermería (INVESTEN)}

El objeto de esta acción es desarrollar una estrategia que organice y facilite la investigación en enfermería. Esta estrategia se fundamenta en fomentar la Integración de la investigación de enfermería en la práctica clínica diaria y como elemento para su desarrollo; en colaborar en el diseño, formulación y ejecución de las políticas para el desarrollo de la investigación en Enfermería; en asesorar, tanto a los profesionales interesados en investigar, como a las agencias o instituciones públicas y privadas que lo soliciten; en realizar formación en Investigación para los profesionales interesados en la investigación en enfermería, en estrecha relación con las estructuras existentes; en difundir los recursos, actividades, bases de datos bibliográficos, resultados de investigación, etc; y en desarrollar las funciones de coordinación desarrollo, agrupando los proyectos (existentes y nuevos) en grupos y líneas de investigación, promocionando el desarrollo de nuevos proyectos en los temas establecidos.

\section{- Institutos de Investigación Sanitaria}

El objetivo de esta acción es desarrollar un sistema de acreditación de Institutos de Investigación Sanitaria considerados como asociaciones de centros de investigación, multidisciplinares y multiinstitucionales, incluidas las estructuras de investigación del Sistema Nacional de Salud, con el fin de desarrollar e integrar armónicamente la investigación básica, clínica y de salud pública como un todo, potenciando la investigación traslacional con una mejor transferencia de los avances científicos obtenidos en la prevención y tratamiento de los problemas de salud más prevalentes. Además, la integración de los distintos tipos de investigación permitirá acortar el intervalo transcurrido entre la producción de un nuevo conocimiento (eficacia) y su transferencia y aplicabilidad real (efectividad y eficiencia) en la practica médica.

Aquellos Institutos de Investigación Sanitaria que resulten acreditados, podrán ser declarados según las actividades que desarrollen, Centros Asociados al Instituto de Salud Carlos III, mediante Convenio, de acuerdo al artículo 4 apartado a) del Estatuto del Instituto de Salud Carlos III, aprobado por Real Decreto $375 / 2001$, de 6 de abril.

Todas estas acciones se deben completar en un futuro, cuando se finalice el mapa de la investigación biomédica, con una acción de apoyo a los Grupos Consolidados, considerados como grupos con calidad, liderazgo y relevancia en sus aportaciones así como por su composición mínima, coherencia, estabilidad y capacidad científica. Se debería realizar una Evaluación externa e independiente de las actividades realizadas en los cinco años anteriores como máximo, completada con una Evaluación in situ de los grupos y asegurar, si procede una financiación a cinco años.

\section{BIBLIOGRAFIA}

1. National Center for Research Resources. A Plan for the National Center for Research Resources: 1998-2003. Bethesda; 1999.

2. Plan Nacional de Investigación Científica, Desarrollo e Innovación Tecnológica, 2004-2007. Ministerio de Ciencia y Tecnología. Noviembre 2003.

3. Buxton, M, Hanney, S. How can payback form health services be assessed? J Healh Serv Res Policy Pre-Iaunch Issue; 1995.

4. Artells Herrero JJ. Estrategia y asignación de recursos en la investigación biomédica. Gac Sanit 2000; 14:391-397.

5. Pellegrini A. Ciencia en pro de la Salud. Publicación Científica y Técnica $\mathrm{N}^{\circ}$ 578. OPS.2000.

6. Ad Hoc Committee on Health Research Relating to Future Intervention Options. 1996. Investing in health research and development. Geneva: World Health Organization. (Document TDR/Gen/96.1).

7. Advisory Committee on Health Research 1998. A research policy agenda for science and technology to support global health development. Geneva: World Health Organization (Document WHO/RPS/ACHR/98.1).

8. Sánchez D, Bazzani R, Gómez S, eds. 1998. Prioridades en la 
investigación de la salud colectiva en América Latina. Montevideo: GEOPS, Trilce.

9. Research and The Governmet Performance and Results Act. COSESUP. USA 1999

10. Peiró S, Artells Herrero JJ. La gestión de la Investigación en los Centros Sanitarios. Una exploración mediante la técnica de grupo nominal. Gaceta Sanitaria 2001; 15: 245-250.

11. Linnako E. Cost and reimbursement of medical teaching and clinical research in Finland. World Hospital and Health Services 1997;33(3):16-20.

12. Linna M, Häkkinen U, Linnako E. An econometric study of cost of teaching and research in Finnish hospitals. Health Economics 1998;7:291-305.

13. Badía X. La investigación de resultados en salud. Barcelona. Edimac, 2000.

14. Segura A. La Influencia de la Investigación en las Políticas de Salud y en la Práctica Sanitaria.. Revista Española de Salud Pública 2000; 74:87-93.

15. Smith R. Measuring the social impact of research. BMJ 2001;323:528.

16. Health Sciences Subcommittee of the Medical Committee of Royal Netherlands Academy of Arts and Sciences. The societal impact of applied health research : towards a quality assessment system. Amsterdam: KNAW, 2001 www.knaw.nl/cg.

17. Orden SCO/2023/2003, de 10 de julio por la que se convocan ayudas para el programa de Recursos Humanos y Difusión de la Investigación Biomédica del Instituto de Salud Carlos III para el año 2003 (BOE de 18/07/2003).

18. Orden SCO/1816/2002 por la que se convocan becas del Fondo de Investigación Sanitaria / Fulbright de ampliación de estudios en los Estados Unidos de América para titulados superiores españoles del programa de recursos humanos y difusión de la investigación biomédica del Instituto de Salud Carlos III (BOE de 16/7/2002).

19. Orden SCO/2929/2003, de 20 de octubre, por la que se convocan ayudas para contratos de investigadores en el Sistema Nacional de Salud, dentro del Programa de Recursos Humanos y Difusión de la Investigación del Instituto de Salud "Carlos III" (Ministerio de Sanidad y Consumo) en el marco del Programa Nacional de Potenciación de Recursos Humanos del Plan Nacional de I+D+I 2000-2003 (BOE de 22/10/2003).
20. Orden de 10 de septiembre de 2001 por la que se crea y regula un nuevo Fichero de Investigadores del Sistema Nacional de Salud con datos de carácter personal, gestionado por el Ministerio de Sanidad y Consumo (BOE de 20/9/2001).

21. Orden de 4 de octubre de 2001 por la que se convocan ayudas para la realización de contratos de apoyo a la investigación en el Sistema Nacional de Salud, dentro del Programa de Recursos Humanos y Difusión de la Investigación del Instituto de Salud Carlos III (Ministerio de Sanidad y Consumo), en el marco del Programa Nacional de Potenciación de Recursos Humanos del Plan Nacional de I+D+I 2000-2003 (BOE de 7/11/2001).

22. Orden SCO/1620/2003, de 3 de junio, por la que se convocan ayudas, para contratar profesionales sanitarios que hayan finalizado el período de Formación Sanitaria Especializada, dentro del Programa de Recursos Humanos y Difusión de la Investigación del Instituto de Salud Carlos III, en el marco del Programa Nacional de Potenciación de Recursos Humanos del Plan Nacional de I+D+I 2000-2003 (BOE de 19/06/2003).

23. Orden SCO/3425/2002, de 20 de diciembre, por la que se convocan ayudas del Programa de Promoción de la Investigación Biomédica y en Ciencias de la Salud del Ministerio de Sanidad y Consumo, para la realización de proyectos de investigación en el marco del Plan Nacional de I+D+I 2000-2003 (BOE de 9/1/2003).

24. Orden SCO/2901/2003, de 10 de octubre, por la que se establecen las bases y se convocan ayudas económicas del Programa de Fomento de la Investigación Biomédica y en Ciencias de la Salud del Instituto de Salud Carlos III, con el fin de dotar de infraestructuras a los centros del Sistema Nacional de Salud en el marco de actuaciones del Plan Nacional de Investigación Científica, Desarrollo e Innovación Tecnológica 2000-2003 (BOE de 21/10/2003).

25. Orden SCO/2765/2003 de 19 de septiembre por la que se convocan ayudas económicas del programa de Promoción y Fomento de la Investigación Biomédica y en Ciencias de la Salud del Instituto de Salud Carlos III, con el fin de realizar estudios e investigaciones sobre evaluación de tecnologías sanitarias (BOE de 8/10/2003).

26. Orden SCO/709/2002, de 22 de marzo de 2002 por la que se convoca la concesión de ayudas para el desarrollo de Redes Temáticas de Investigación Cooperativa (BOE de 3/4/2002). 